\title{
Timing-Based Model of Body Schema Adaptation and its Role in Perception and Tool Use: A Robot Case Study
}

\author{
Cota NABESHIMA, Max LUNGARELLA and Yasuo KUNIYOSHI \\ Intelligent Systems \& Informatics Laboratory \\ Department of Mechano-Informatics, Graduate School of Information Science \& Technology \\ The University of Tokyo, Japan \\ \{nabesima, maxl, kuniyosh\}@isi.imi.i.u-tokyo.ac.jp
}

\begin{abstract}
The multisensory representation of our body (body schema), and its conscious and manipulable counterpart (body image) play a pivotal role in the development and expression of many higher level cognitive functions, such as tool use, imitation, spatial perception, and self-awareness. This paper addresses the issue of how the body schema changes as a result of tool use-dependent experience. Although it is plausible to assume that such an alteration is inevitable, the mechanisms underlying such plasticity have yet to be clarified. To tackle the problem, we propose a novel model of body schema adaptation which we instantiate in a tool using robot. Our experimental results confirm the validity of our model. They also show that timing is a particularly important feature of our model because it supports the integration of visual, tactile, and proprioceptive sensory information. We hope that the approach exposed in this study will allow to gain further insights into the development of tool use skills and its relationship to body schema plasticity.
\end{abstract}

Index Terms-Adaptive body schema, Extensible body image, Tool-using robot, Time-domain visual-tactile integration, Developmental robotics

\section{INTRODUCTION}

Robots intended to interact with human beings require high-level cognitive capabilities. In humans (as well as other animals) many of those capabilities are based on an internal representation of the body. Two distinct and complementary definitions of such representation exist [1]: (a) the body schema, that is, an unconscious neural map of the spatial relations among the parts of the body, in which multi-modal sensory information (e.g. visual, somatosensory, and tactile) is integrated [2], [3]; and (b) the body image, a consciously manipulable and body-centered version of the body schema [4]. In this paper, we will mainly consider the first definition.

Many fundamental abilities rely on the availability of a representation of the body. Humans, for instance, imitate other people's actions by projecting the observed behavior onto their own bodily possibilities. This process occurs at an unconscious level, and is known as "transfer" of the body schema [5]. Representations of the body also relate to the subject's perceptual experience of her/his own body (self-awareness), and of her/his conceptual understanding of the body in general [6]. Interestingly, the functional requirement for a body schema extends even beyond the boundary set by our body. To guide movements through space, for instance, the brain must constantly monitor limb position and body movement in relation to objects contained in the body's peripersonal space (that is, the space immediately surrounding the body) [7]. In addition, the sensory information from the peripersonal space is not only visual and auditory but also tactile and proprioceptive [3]. It follows that also spatial perception relies on the body schema. A similar state of things holds for tool use. Neurophysiological evidence shows that by repeatedly using a tool, changes in the body representations (of humans and trained monkeys) occur, and over time the tool is felt as being an integral part of the body. The phenomenon describing the extension of the body image (or the body schema for that matter) to incorporate noncorporeal objects, such as tools, has been reported in the literature and is known as body image or body schema extension [8].

The core assumption of the work presented here is that the body schema plays a significant role in constructing a robotic system capable of realizing the aforementioned higher-level functionalities. Unfortunately, to date the mechanisms underlying body schema acquisition and plasticity are not yet completely understood. In this paper, we tackle the problem by addressing it through the lens of developmental robotics [9]. Our approach is synthetic: we first attempt to extract principles from neurophysiological and neuropsychological findings, and then employ those principles to realize higher level functionalities in artificial systems. The principle used in this paper is unconventional and relies on the concept of causality: the body representation (here: body schema) of the actor (trained monkey, human subject, or robot) extends if the temporal causal relationship among the sensory modalities matches a relationship the actor has previously experienced before. The high-level function studied is tool use. We hypothesize that because tool use typically improves object manipulation abilities, the effect of body schema alteration will be easier to detect and understand.

In the following sections, we first briefly describe the notion of body schema. We then present a novel model of body schema adaptation, and instantiate it in a real robot which has to learn to use a tool. We present some results confirming the validity of our approach. Before concluding, we discuss how our model may be useful to understand how tools are actually incorporated into the body schema of an actor interacting with its local environment. 


\section{PERSPECTIVES ON BODY SCHEMA}

In this section, we present a view on the body schema which relies on findings from the areas of neurophysiology and neuropsychology. We then briefly survey some previous research applying the concept of body schema to robots.

\section{A. Body schema and phantoms}

The term body schema was initially introduced by Head and Holmes [2] to describe (a) the mapping from proprioception and efferent copy (copy of motor command) to body posture and motion, and (b) the mapping from tactile sensation to its originating position on the body surface. We call this original concept the "geometric body schema." The concept of body schema has been substantially enriched by the discovery of the phenomenon of phantom limbs [10]. The phenomenon can be observed in amputees who find themselves feeling somatic sensations (such as pain, tickle, or itch) originating from their amputated (that is, inexistent) phantom limbs. Interestingly, a subsequent study showed that phantom limbs do not occur in case of congenitally missing limbs [11]. One possible implication of these facts is that the body schema is (a) formed during development (hence the absence of phantoms in people born without a limb), and (b) static once acquired. Indeed, throughout development, we experience a flood of multisensory information, and the body schema might simply be the result of how sensory feedback is organized and integrated.

\section{B. Body schema and plasticity}

Recent studies shed new light on the concept of body schema. Phantom limbs, for instance, can be treated with mirrors which give amputees relief from the disturbing presence of the lost limb [12]. This phenomenon demonstrates that the body schema is alterable even in adults. The rationalization of the discrepancy between visual and phantom somatosensory information induces them to "accept" to the new body. As a consequence of this recent body of work, the body schema is nowadays considered being plastic and constantly updated by sensory feedback (e.g. [13]). Iriki et al. reported the existence of a class of bimodal neurons in the monkey's intraparietal cortex activated by somatosensory and visual stimuli from the monkey's hand [8]. They were able to show that a visual stimulus approaching the tip of a rake was sufficient to activate the bimodal neurons of monkeys trained to retrieve distant objects using that tool. This finding confirms the hypothesis that the body schema actually extends to incorporate tools and other objects.

Some other remarkable examples of body schema extension are described in the literature. An example is schematically represented in Fig.1 (see also [14]). In this particular experiment, a subject sits in front of a table with his/her hands hidden under the table. Now, the experimenter simultaneously either strikes or taps the subject's hand and an unreachable point on the table. After a minute or so, the subject starts feeling a stroke on his/her hand as thought

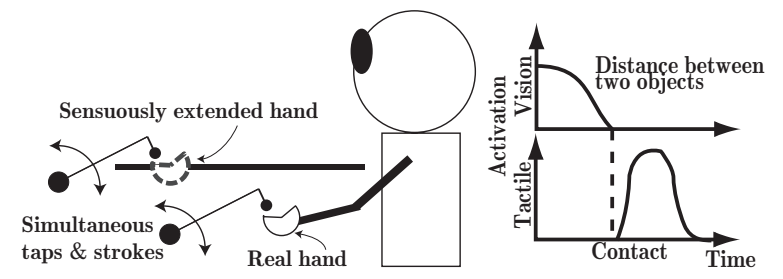

Fig. 1. Schematic representation of the phenomenon of body image extension. If the taps and strokes are simultaneous, then the subject feels the table as being part of her/his body.

it emanates from the point on the table (despite the table being inanimate). This phenomenon is a striking example of "body image extension" and the table is consciously thought to be part of the body. The study provides yet more evidence that body representations such as body schema and body image are plastic and easily alterable (even in adults).

\section{Body schema and robots}

The concept of the body schema is closely related to modeling techniques adopted in traditional robotics. Similar to the geometric body schema, conventional kinematic and kinetic models of the body of the robot are static and geometric. In addition, in robotics, geometric transformations are used to combine different kinds of sensory information: visual and proprioceptive [15], or visual and auditory [16]. By contrast to the conventional approach, some recent research has also attempted to construct plastic body schema in robots. Yoshikawa, for instance, proposed an adaptive body schema acquisition system which uses vision to detect regions of space occupied by the body and to learn a map of the body [17]. Stoytchev constructed a model of body schema extension, and by assuming that it is possible to detect a change of the body, he proposed a method to automatically adapt the kinematic controller [18]. Another related piece of work is the one by Fitzpatrick and Metta who constructed a machine vision system capable of extracting a region of the arm by simply touching an external object [19].

Despite these modeling efforts sort of break with the traditional approach, they neither realize a biologically plausible mechanism of body schema supporting high-level functions, nor do they provide any satisfying explanation of "body image extension." We address both issues in this paper.

\section{ADAPTIVE MODEL OF BODY SCHEMA}

In this section, we introduce a novel model of body schema adaptation which relies on the integration in space and in time of constantly updated multisensory information.

\section{A. Timing-based model}

As already stated in Section II-B, the body schema is not static but changes plastically. Here, we review the phenomenon of "body image extension" (Fig.1), and propose a mechanistic model for it. In this particular phenomenon, the spatio-temporal patterns of visual and tactile sensory activity induce the brain to "think" that the table is a new 
part of the body (an extension). Based on this finding, it is possible to derive a model for body schema extension.

A first assumption is that visual and tactile information are integrated in time and in space. Indeed, visual and tactile sensations are temporally integrated because they are causally related. Moving the hand to approach a target represents the cause, and the tactile sensation (contact) the corresponding effect. This means that by converting visual information (available during the approach phase) into tactile information (resulting from the contact with the target object), it is possible to predict the cause of a possible action. Moreover, tactile and visual information are also spatially integrated: visual "contact" (that is, overlap of hand and target object) and tactile contact occur on the same point on the body. The two modalities are integrated and unified by the event "contact." The second assumption is that the unity of integration is stored in an associative memory so that if one modality is active, the other is also activated. For instance, only if the temporal causality concerning "contact" is the same as the causality experienced, are the two locations from which visual and tactile "contact" information originate regarded as the same location.

\section{B. Tool use model with plastic body schema}

We enhance our model of body schema adaptation to include adaptive tool use. The information flow through our model is depicted in Fig.2. This model enables a robot to reach and touch a target with a tool as though the tool is the robot's hand. Note that knowledge about the tool is a priori unavailable, and the robot autonomously incorporates the tool and learns to use it.

In order to reach the target with its hand, the robot executed the following steps: The robot (a) generates paths from locations of its hand $\boldsymbol{r}_{h}^{V}$ and a target $\boldsymbol{r}_{t}^{V}$, (b) internally simulates the paths and selects an executable one using the kinematic controller of its hand, (c) actually moves the hand along the path using the controller, and (d) reaches and touches the target. The temporal visual information $\boldsymbol{r}_{h}^{V}$ and tactile patterns $\boldsymbol{S}^{T}$ obtained here are learned in an associative memory, which works as a time-domain integrator.

When the robot holds and swings a stick, and accidentally hits a target with its tip, it obtains touch sensation $S^{T}$ relayed by the stick and visual location of the stick

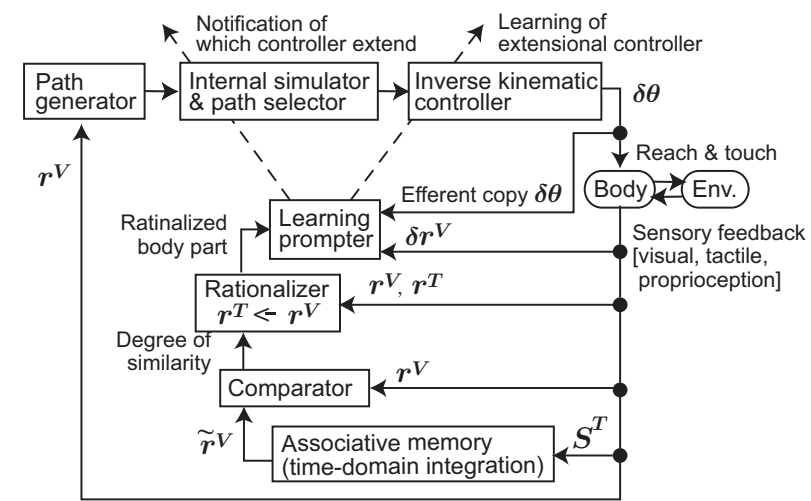

Fig. 2. Model of body schema extension applied to tool use task.
$\boldsymbol{r}_{s}^{V}$. When this $\boldsymbol{S}^{T}$ is fed to the associative memory, the associative memory recalls the learned visual pattern $\tilde{\boldsymbol{r}}_{h}^{V}$. Then, $\tilde{\boldsymbol{r}}_{h}^{V}$ is compared with $\boldsymbol{r}_{s}^{V}$. A similarity between the two patterns invokes spatial rationalization $-\boldsymbol{r}_{s}^{V}$ where visual "contact" occurs is regarded as the location where $\boldsymbol{S}^{T}$ comes, that is, $\boldsymbol{r}^{T}$.

By using this strategy, the robot can determine which controller should be adapted to use the stick (here, the controller of the hand corresponds to that), and to learn the extensional controller using visual information $\boldsymbol{r}_{s}^{V}$ and the efferent copy $\delta \boldsymbol{\theta}$. After learning, the robot is able to use the tool in the same manner as the hand: in the case of the tool, it can generate paths, internally simulate the paths and move the tool along an executable path trajectory. We can say that the stick (that is, the tool) has become part of the body.

\section{IMPLEMENTATION}

In this section, we describe our experimental setup and the implementational details of our model.

\section{A. Experimental setup}

The robot used in our experiments was equipped with one camera, two touch sensors (located in its hand), and a two-link manipulator constrained to move in a plane. The tool is an extension of the distal link of the manipulator. The information entering the system was the following: (a) tactile information $\boldsymbol{S}^{T}$ is the signal pattern originating from the touch sensors; (b) visual information consists of the planar positions of the robot's hand $\boldsymbol{r}_{h}^{V}$, the tip of a stick $\boldsymbol{r}_{s}^{V}$ or a target object $\boldsymbol{r}_{t}^{V}$; and (c) proprioceptive information $\boldsymbol{\theta}$ is given by the joint angles.

We constructed a robotic system composed of the upper body of a small-sized humanoid robot and a color CCDcamera (Fig. 3). The hand of the robot held a stick, so that a contact between the stick and a distant object activated the switches in the hand's palm. Note that the activation of the switches was dependent on the target approach direction. For the acquisition of the positions of limbs and objects on the plane and in order to pre-calibrate the camera-centered coordinate system, we used a set of perspective images collected with the CCD-camera before performing the actual experiments. The Jacobian matrix and the forward kinematic equations were given only for the hand.



Fig. 3. Left: Robot used in our experiments. Right: Snapshots of reaching experiment. 


\section{B. Design of spatio-temporal associative memory}

The associative memory required by our model is visualized in Fig.4. It consisted of the combination of two associative memories: (a) a gating neural network (GNN) which associated visually detected target approach direction with tactile information, and (b) a nonmonotone neural network (NNN) [20] which temporally associated tactile signals with a visual distance between the hand and a target. Both networks were essentially augmented Hopfield neural networks.

The GNN had twelve neurons whose activation function was,

$$
y_{i}=\operatorname{sgn}\left(\sum_{j} w_{i j} y_{j}+z_{i}-\mu\right)
$$

where $y_{i}$ is the output of neuron $i, z_{i}$ the input to neuron $i$, $w_{i j}$ the connection weights from neuron $j$ to neuron $i, \mu$ a given threshold, and $\operatorname{sgn}(x)$ returns +1 or -1 according to the sign of its argument $x$. The GNN obeyed an augmented Hebbian learning rule [21]:

$$
w_{i j}=w_{i j}+y_{i}\left(y_{j}-y_{i} w_{i j}\right)
$$

As shown in Fig.4 (middle), the tactile feedback $\boldsymbol{S}^{T}$ was fed to two neurons. The approaching direction $\phi$ of two objects was weighed by ten Gaussian kernels fully connected to each other. The purpose of the GNN was to associate tactile sensory channels with visual information about the object approach directions.

The outputs of the tactile neurons of the GNN were eventually summed according to:

$$
p=\sum_{t} \sum_{i \in \text { tactile }} y_{i} \cdot \Delta t
$$

where $p$ is the sum of the output neurons of the GNN. Note that the purpose of this sum is to transform tactile patterns $\boldsymbol{S}^{T}$ into a wave form with a slope, reason being that it is easier for the NNN to learn patterns containing slopes.

The NNN was composed of 40 neurons. As shown in Fig.4 (bottom), 20 neurons of the NNN coded for the distance $d(t)$ of the two objects, whereas the other 20 neurons coded for the tactile pattern $p(t)$. Both were weighed by twenty Gaussian kernels in the same way as $\phi$.

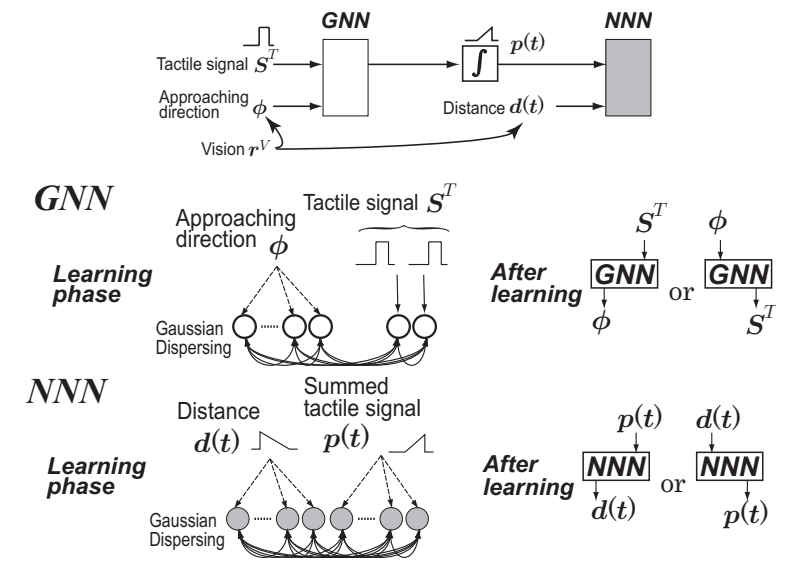

Fig. 4. Visuo-tactile associative memory for spatio-temporal patterns. The memory consists of a gating neural network (GNN) and a nonmonotone neural network (NNN).
The dynamics of the NNN was governed by the following set of equations:

$$
\begin{aligned}
\tau \frac{d u_{i}}{d t} & =-u_{i}+\sum_{j} w_{i j} y_{j}+z_{i} \\
y_{i} & =f\left(u_{i}\right) \\
f(x) & =\frac{\left(1.0-e^{-c x}\right)\left(1.0+\kappa e^{c^{\prime}(|x|-h)}\right)}{\left(1.0+e^{-c x}\right)\left(1.0+e^{c^{\prime}(|x|-h)}\right)}
\end{aligned}
$$

where $\tau$ is an update time constant, $u_{i}$ the internal potential of a neuron $i, w_{i j}$ the connecting weight from a neuron $j$ to a neuron $i, z_{i}$ the external input to neuron $i$, and $y_{i}$ the output of neuron $i$ calculated by a nonmonotone output function (6). The learning rule of the NNN was,

$$
\tau^{\prime} \frac{d w_{i j}}{d t}=-w_{i j}+\alpha \gamma_{i} y_{i}
$$

$\tau^{\prime}$ being the learning time constant, $\alpha$ the learning coefficient, and $\gamma_{i}$ the learning signal for a neuron $i$. We determined $\alpha=\alpha^{\prime} x_{i} y_{i}$ with a constant $\alpha^{\prime}$ and $z_{i}=\lambda \gamma_{i}$ in the same way as described in the original paper by Morita [20]. For all neurons $\lambda$ was constant throughout the learning phase in order to realize a bi-directional associative memory.

These coupled networks could effectively learn the association among spatio-temporal patterns. Because of Hebbian-like learning, the tactile and visual patterns were just fed to the networks during the learning phase. Due to its associative capabilities, when one modality was fed to the system, the pattern of the other modality could be recalled.

\section{Design of kinematics learning}

In our experiments the tool was a stick attached to the distal link of the arm of the robot. Our system successfully learned a kinematic controller for tool use. The relation of joint angles $\boldsymbol{\theta}$ and the position of the end effector $\boldsymbol{r}$ were given by:

$$
\begin{aligned}
\delta \boldsymbol{r} & =\boldsymbol{J}(\boldsymbol{\theta}) \delta \boldsymbol{\theta} \\
\boldsymbol{J}(\theta) & =\left[\begin{array}{cc}
-l_{1} s_{1}-l_{2} s_{12} & -l_{2} s_{12} \\
l_{1} c_{1}+l_{2} c_{12} & l_{2} c_{12}
\end{array}\right]
\end{aligned}
$$

where $J$ is a Jacobian matrix, $l_{j}(j=1,2)$ is the length of link $i, s_{1}$ is $\sin \theta_{1}, c_{1}$ is $\cos \theta_{1}, s_{12}$ is $\sin \left(\theta_{1}+\theta_{2}\right)$, and $c_{12}$ is $\cos \left(\theta_{1}+\theta_{2}\right)$.

The following equations were applied to the tip of the hand and of a stick:

$$
\begin{aligned}
\delta \boldsymbol{r}_{h} & =\boldsymbol{J}_{h}(\boldsymbol{\theta}) \delta \boldsymbol{\theta} \\
\delta \boldsymbol{r}_{s} & =\boldsymbol{J}_{s}(\boldsymbol{\theta}) \delta \boldsymbol{\theta}
\end{aligned}
$$

where $\boldsymbol{r}_{h}$ and $\boldsymbol{J}_{h}$ are the position and the Jacobian matrix of the hand, and $\boldsymbol{r}_{s}$ and $\boldsymbol{J}_{s}$ are those of the stick.

Note that the only difference between hand and stick was their length. Having a stick turns $\boldsymbol{J}_{h}$ with a constant matrix $\boldsymbol{J}_{c}$,

$$
\boldsymbol{J}_{s}(\boldsymbol{\theta})=\boldsymbol{J}_{h}(\boldsymbol{\theta}) \boldsymbol{J}_{c}
$$


It is almost trivial for Jacobian matrices of the form (9). Equation (12) means that a robot can use a stick with a brief transformation. Here, we substitute (12) into (11) and rearrange the equation with $\boldsymbol{J}_{h}(\boldsymbol{\theta}[t])$ and the displacement $\delta r_{s}[t]$ made by an actual motion $\delta \boldsymbol{\theta}[t]$,

$$
\begin{aligned}
\boldsymbol{\Theta}^{\prime} & =\boldsymbol{J}_{c} \boldsymbol{\Theta} \\
\boldsymbol{J}_{c} & =\boldsymbol{\Theta}^{\prime} \boldsymbol{\Theta}^{+} \\
\boldsymbol{\Theta}^{\prime} & =\left[\begin{array}{lll}
\left(\boldsymbol{J}_{h}^{-1}(\boldsymbol{\theta}) \delta \boldsymbol{r}_{s}\right)[t] & \left(\boldsymbol{J}_{h}^{-1}(\boldsymbol{\theta}) \delta \boldsymbol{r}_{s}\right)[t+1] & \ldots
\end{array}\right] \\
\boldsymbol{\Theta} & =\left[\begin{array}{lll}
\delta \boldsymbol{\theta}[t] & \delta \boldsymbol{\theta}[t+1] & \ldots
\end{array}\right]
\end{aligned}
$$

with + is the pseudo-inverse symbol.

These equations indicate that the constant matrix $\boldsymbol{J}_{c}$ is learnable through experience and should be relatively easy to converge to because it is linear and constant. We implemented the incremental learning of this matrix with a vanilla-type two-layered perceptron whose input and output were $\boldsymbol{J}_{h}^{-1}(\boldsymbol{\theta}) \delta \boldsymbol{r}_{s}$ and $\delta \boldsymbol{\theta}$ respectively. This perceptron worked as the extensional transformation $\boldsymbol{J}_{c}^{-1}$ for the controller of the hand $\boldsymbol{J}_{h}^{-1}$.

\section{EXPERIMENTS}

In our experiments, the robot reached and touched a target with its hand, and consequently experienced multimodal sensory information. Such information was integrated spatially and temporally. If the robot accidentally hit a target and the resulting sensory feedback was temporally consistent with the stored integration, the robotic system became "aware" of the extension of its hand and began learning an extensional controller for the stick. After a while, the robot learned to use the stick as a tool in the same manner as its hand.

To generate the reaching movements, we used quadratic Bezier curves describing the path connecting hand and target. The curves reached the target by approaching the it from four different directions (60, 90, 240, 270 degree). The robot internally simulated those movements, chose a possible motion in the condition of joint limit, and then eventually executed the movement as shown in Fig.3 (right). The expression of this behavior led a set of visualtactile patterns: the cases of 90 and 270 degree are shown in 5 (left), whereas the case of 60 and 240 degree are displayed in Fig. 5 (right). The top of the figure is the distance $d$ between the target and the hand. Each waveform shows an attempt to approach the target. The second top is the direction $\phi$ from which the hand approaches the target. The two bottoms are the response patterns of tactile sensors $A$ and $B$ located in the palm of the hand. The patterns were aligned in time so that the time of contact was 0 [msec]. Because the visual patterns were cluttered after contact, they were delayed by 1500 [msec], and then fed as learning signals to the augmented associative memory. The NNN updated its activation patterns until its state stabilized for every input at each time step. The parameters of the NNN are listed in Table I. Similarly, the GNN learned at every time step the spatio-temporal patterns of its input.

Next, we made the robot hold and swing a stick. When the robot accidentally hit the target with the tip of the stick,

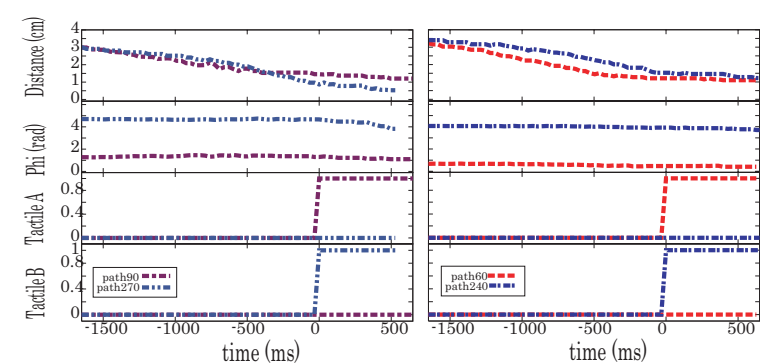

Fig. 5. Spatio-temporal patterns of visual and tactile sensors generated when reaching the target from different directions. Left: 90 and 270 degrees, right: 60 and 240 degrees.

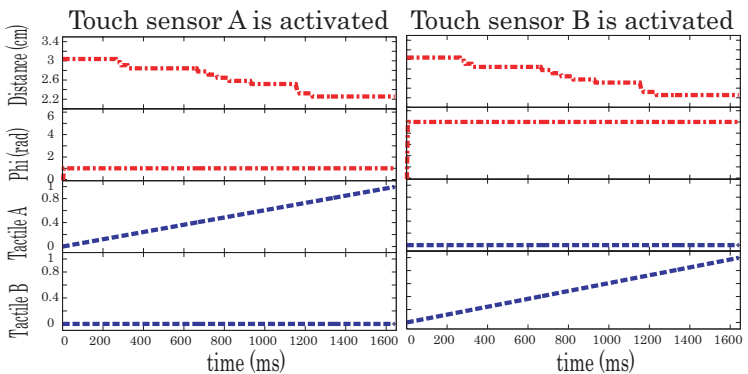

Fig. 6. Visual patterns recalled by the associative memory when tactile pattern is used as input. Left: input is activation of touch sensor A. Right: input is activation of touch sensor B. The tactile patterns are timeintegrated.

it obtained concurrent tactile and visual information. The consequent tactile activation patterns was then fed to the associative memory, and the visual patterns $d$ and $\phi$ were extracted from the NNN through the GNN.

Two input and output patterns are shown in Fig.6. One corresponds to the activation of one tactile sensor, whereas the other relates to the activation of the other tactile sensor. The recalled patterns were time-correlated with the visual patterns obtained when the robot swang a stick and hit the target. The temporal consistency and spatial difference between the hand and the tip of the tool drove the system to learn the Jacobian matrix transformation.

For adaptive learning of the controller, the robot moved its joints individually (as shown in Fig. 7 (left) and Fig. 8 ), and the obtained visual patterns $\delta r_{s}$ as well as the perceived movement $\delta \boldsymbol{\theta}[t]$ were fed every 500 [msec] to the perceptron 50 times with a learning coefficient $D=10$.

The learning converged rapidly as depicted in Fig. 7 (right). Our results $\boldsymbol{J}_{c}$ were in agreement with one significant figure with the value theoretically calculated by

TABLE I

PARAMETERS FOR NONMONOTONE NEURAL NETWORK. $\begin{array}{ccccccccc}\Delta t & \tau & \tau^{\prime} & \lambda & \alpha^{\prime} & c & c^{\prime} & h & \kappa\end{array}$

Learning of kinematics transformation
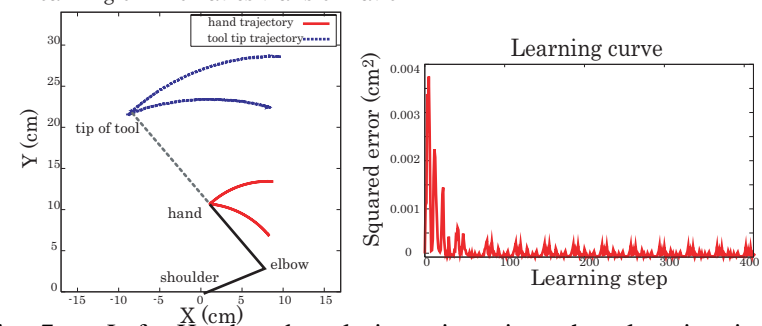

Fig. 7. Left: Hand and tool tip trajectories when learning inverse kinematic transformation. Right: Learning curve. 


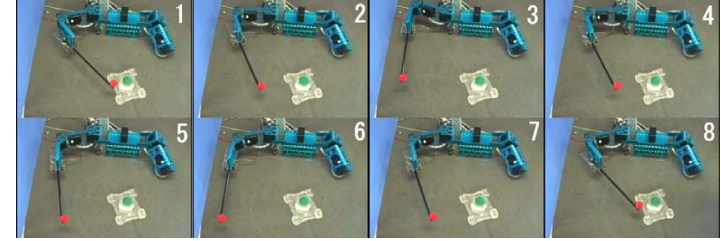

Fig. 8. Experiment of inverse kinematics learning.

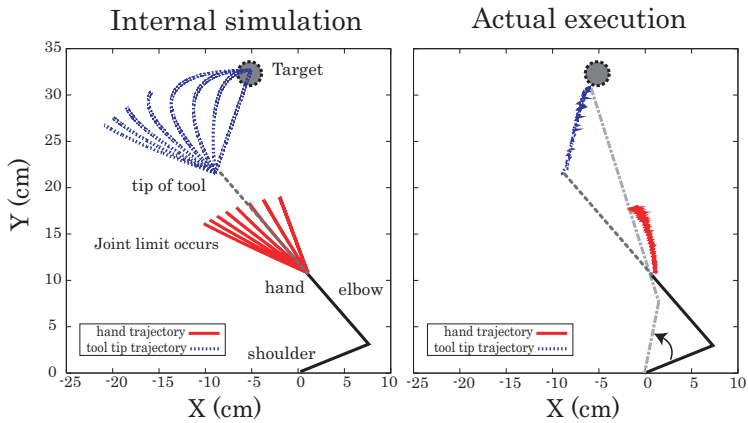

Fig. 9. Reaching a target unreachable without a tool. Left: internally simulated trajectories. Right: actually performed movement.

\section{(12) for a given $\boldsymbol{J}_{s}$.}

Eventually, the robot learned to use the stick like the hand with the learned transformation $\boldsymbol{J}_{c}$. As for the hand, by using quadratic Bezier curves obtained from the hand were used to generate reaching movements. The movements were first simulated internally, and after chosing a possible motion pattern, actually executed.

The simulated trajectories are reproduced in Fig. 9 left. The lines ending halfway indicate rejection at the ending points due to the joint limit. The lines that reach the target give paths that can be used to perform a movement. The robot was able to judge whether the target is reachable by the hand or by the stick. Here, it could decide to use a tool and execute one of the possible paths. The trajectories of the hand and stick tip are depicted in the right of Fig. 9. Because of the volume of the target object, this trajectory ended in front of the center of the target when the stick touched the target object.

\section{CONCLUSION}

In this paper, we proposed a novel model of body schema adaptation. Our model was inspired by neurophysiological findings, in particular a phenomenon known as "body image extension", which has been observed in monkeys and in humans. We tested our model by instantiating it in a robot whose task was to learn to use a simple tool. Our experimental results show that the model allows the robot to extend its body schema to incorporate an external (extracorporeal) object through the time-domain integration of multisensory information (tactile and visual). We conclude that body schema adaptation might actually be a good strategy to learn to use tools through actor-environment interaction. Another conclusion is that a plastic body schema is a necessary requirement for tool use skills to emerge, and that the approach in which first a body schema is constructed may have actual validity for achieving higher level functions.

In line with the approach advocated by developmental robotics, our model represents only the first step towards the acquisition of a truly adaptive robotic body schema. If a robot could acquire a body representation similar to ours, then it might actually also develop high level cognitive functions such as imitation [5], [6] or spatial perception [7]. Future work will be mainly aimed at extending the model proposed in this paper to include imitative learning.

\section{ACKNOWLEDGMENTS}

This study was supported by the Advanced and Innovational Research program in Life Sciences from the Ministry of Education, Culture, Sports, Science and Technology, Japan. The authors would also like to thank the Jap. Soc. for Promotion of Science for a Grant-in-Aid for Scientific Research.

\section{REFERENCES}

[1] S. Gallagher, "Body Image and Body Schema: A Conceptual Clarification," J. of Mind and Behavior, Vol. 7, pp. 541-554, 1986.

[2] H. Head and G. Holmes, "Sensory disturbances from cerebral lesions," Brain, Vol. 34, pp. 102-245, 1911.

[3] N. P. Holmes and C. Spence, "The body schema and multisensory representation(s) of peripersonal space," Cognitive Processing, Vol. 5, No. 2, pp. 94-105, 2004.

[4] P. Schilder, "The Image and Appearance of the Human Body: Studies in the Constructive Energies of the Psyche," Kegan Paul, London, 1935.

[5] M. Merleau-Ponty, "Phénoménologie de la perception," Gallimard, Paris, 1945.

[6] S. Gallagher and A. Meltzoff, "The Earliest Sense of Self and Others: Merleau-Ponty and Recent Developmental Studies," Philosophical Psychology, Vol. 9, pp. 213-236, 1996.

[7] G. Rizzolatti, L. Fadiga, V. Fogassi and V. Gallese, "The space around us," Science, Vol 277(5323), pp. 190-191, 1997.

[8] A. Iriki, M. Tanaka and Y. Iwamura, "Coding of modified body schema during tool use by macaque postcentral neurones," Cognitive Neuroscience \& Neuropsychology, Vol. 7, No. 14, pp. 2325-2330, 1996.

[9] M. Lungarella, G. Metta, R. Pfeifer and G. Sandini, "Developmental robotics: a survey," Connection Science, Vol. 15, No. 4, pp. 151-190, 2003.

[10] M. L. Simmel, "The conditions of occurrence of phantom limbs," Proc. of the American Philosophical Society, Vol. 102, pp. 492-500, 1958.

[11] M. L. Simmel, "The absence of phantoms for congenitally missing limbs," Am. J. of Psychology, Vol. 74, pp. 467-470, 1961.

[12] V. S. Ramachandran and D. Rogers-Ramachandran, "Synaesthesia in phantom limbs induced with mirrors," Proc. of the Royal Society of London, Vol. 263, pp. 377-386, 1996.

[13] A. Maravita and A. Iriki, "Tools for the body (schema)," Trends in Cognitive Sciences, Vol. 8, No. 2, pp. 79-86, 2004.

[14] V. S. Ramachandran and S. Blakeslee, "Phantoms in the Brain: Probing the Mysteries of the Human Mind," Harpercollins, 1998.

[15] S. Ganapathy, "Decomposition of transformation matrices for robot vision," Proc. of Int. Conf. on Robotics and Automation, Vol. 1, pp.130-139, 1984.

[16] Y. Nakagawa, H. G. Okuno and H. kitano, "Using vision to improve sound source separation," Proc. of Nat. Conf. on Artificial Intelligence, pp.768-775, 1999.

[17] Y. Yoshikawa, "Subjective Robot Imitation by Finding Invariance," $\mathrm{Ph} . \mathrm{D}$. thesis, Osaka university, 2005.

[18] A. Stoytchev, "Computational Model for an Extendable Robot Body Schema," Georgia Institute of Technology, College of Computing Technical Report, GIT-CC-03-44, 2003.

[19] P. Fitzpatrick and G. Metta, "Toward manipulation-driven vision", Proc. of Int. Conf. on Intelligent Robot and Systems, 2002.

[20] M. Morita, "Memory and Learning of Sequential Patterns by Nonmonotone Neural Networks," Neural Networks, Vol. 9, No. 8, pp.1477-1489, 1996.

[21] E. Oja, "A Simplified Neuron Model As a Principal Component Analyzer," J. of Math. Biol., No. 15, pp.267-273, 1982. 